\title{
A New Exposition on Cell Division
}

\author{
Ronald L. Huston \\ Professor Emeritus, Mechanical and Materials Engineering, University of Cincinnati, Cincinnati, OH, USA \\ Email: hustonrl@ucmail.uc.edu
}

How to cite this paper: Huston, R.L. (2019) A New Exposition on Cell Division. Advances in Bioscience and Biotechnology, 10, 218-225.

https://doi.org/10.4236/abb.2019.108016

Received: June 24, 2019

Accepted: August 13, 2019

Published: August 16, 2019

Copyright (c) 2019 by author(s) and Scientific Research Publishing Inc. This work is licensed under the Creative Commons Attribution International License (CC BY 4.0).

http://creativecommons.org/licenses/by/4.0/

\begin{abstract}
This paper provides summary description of the procedures by which human and animal cells (eucaryotic cells) divide into two identical parts. The focus is on the nucleus, with particular attention given to the centrosome and the chromosome. Within the centrosome is a pair of organelle known as centriole. When the cell is about to divide, the centrioles duplicate themselves. At the same time, the DNA within the chromosome duplicates itself. The centriole pair, now two pairs, then separate with one pair migrating about the nucleus to the diametrically opposite side. The original and migrated centriole then emit long strands known as microtubules across the nucleus. Similarly, the chromosome emits long strands known as kinetochores. The microtubules and the kinetochores are perpendicular to each other and they cover the nucleus with a checkered appearance. The diametrically opposed centriole then forms centrosomes which pull the nucleus apart. The two nuclear parts then separate with each part taking with its half of the remainder of the cell (the cytoplasm) and thus two virtually identical cells are attained. The significance of this paper is that it provides the reader with a condensed summary of the life-dependent process known as cell division.
\end{abstract}

\section{Keywords}

Cell Division, Nucleus, Centrosome, Chromosome

\section{Introduction}

Cell division and then duplication is vital to the survivability of human and animal ("eucaryotic" beings). This is an accepted fact. Historically, however, there was a time when even the existence of cells was unknown. Indeed, the first signs of cells occurred with the availability of magnifying glasses and later microscopes.

Today, visualization is becoming increasingly sophisticated with the use of ever-advancing electronic technologies. We can now see into the minute geome- 
try of the nucleus. As with many of these findings and discoveries, more questions are then raised than answered.

The balance of this paper is divided into eight sections with the first of this pertaining to aspects of cell geometry. They describe centrioles, the centrosomes and kinetochores. The last three sections describe mitosis, the nucleus changes, and the division of the cytoplasm - the remainder of the cell aside from the nucleus. The final section also provides concluding remarks.

\section{Cell Geometry}

Figure 1 shows a cross-section sketch of a human cell. References [1] [2] [3] are good sources for overall cellular description.

Two items in the sketch are needed for our purposes: the centriole and the nucleus-key players in cell division. The following section describes the centriole and auxiliary tissues.

\section{Centrioles and the Centrosome}

Figure 2 provides a gross representation of the centrioles. From afar, they appear to be perpendicular hollow cylinders, approximately 200 to $300 \mu \mathrm{m}$ in diameter and 400 to $500 \mu \mathrm{m}$ long with a wall thickness of approximately $10 \mu \mathrm{m}$.

A closer look, however, shows that the centriole walls are comprised of nine uncovered (no membrane) "blades" of adjoining microtubules triplets. The following section describes microtubules.

With the centriole occurring in pairs and with perpendicular intersecting axes, one of the centrioles is slightly longer than the other with the shorter one attached to the base of the longer one. The geometry leads these to be known as "mother" and "daughter" centrioles. See Figure 2 and Reference [4]. Reference [4] and its references provide additional details about centrioles.

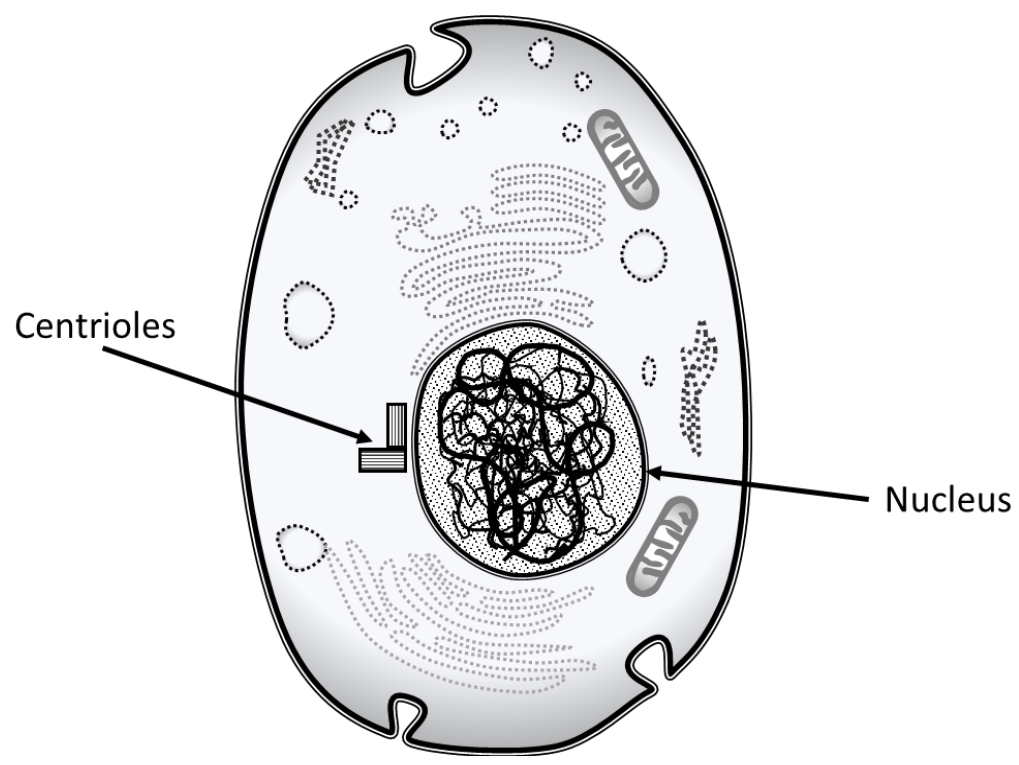

Figure 1. A cross-section sketch of a typical human cell. 


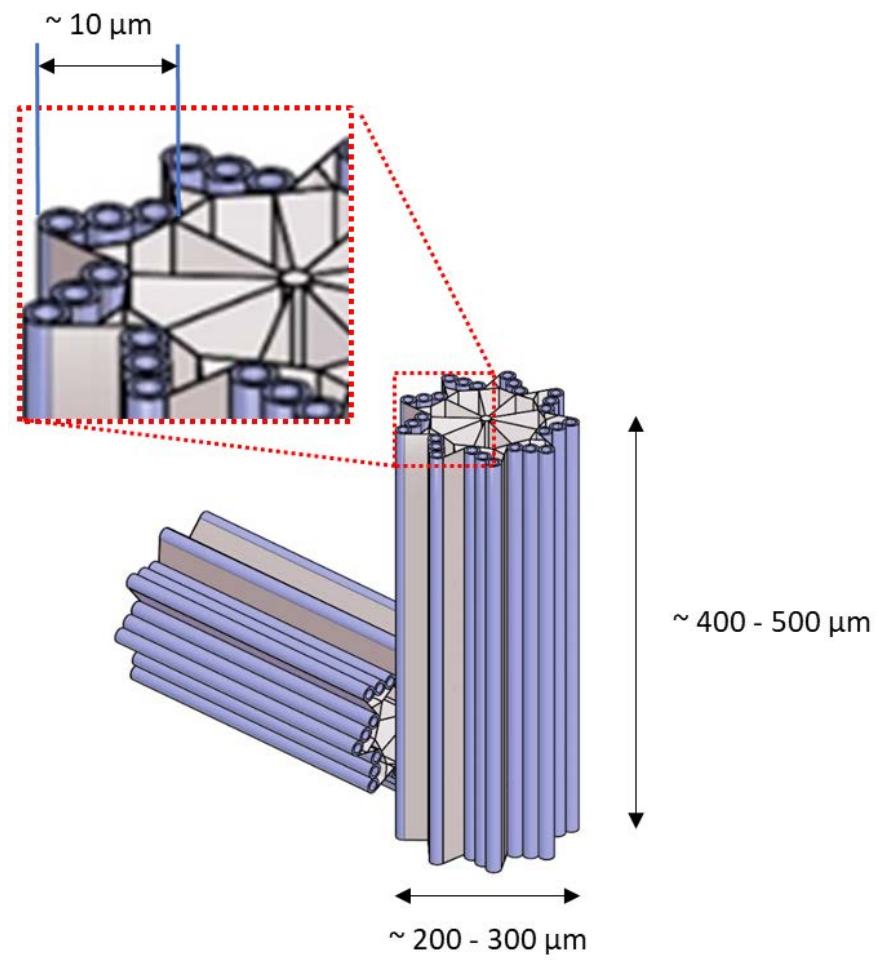

Figure 2. A gross representation of a centriole pair.

The centrioles are immersed in many proteins. The proteins are layered about the centriole forming a mound known as the "centrosome". The centrosome is the source for the development of microtubules. Consequently, the centrosome is also known as the microtubule organizing center (MTOC).

\section{Microtubules}

Reference [5] provides a summary description of microtubules. Figure 3 presents a model of a microtubule.

Microtubules are the most dynamic of all cellular organelles. They are hollow cylinders with $25 \mu \mathrm{m}$ outside diameter, $15 \mu \mathrm{m}$ inside diameter, and varying indefinite length, long enough to extend over half a cell length.

The microtubule walls consist of $\alpha$ and $\beta$ tubulin. Tubulin is a protein with a variety of forms. For structural modelling purposes $\alpha$ and $\beta$ tubulin may be represented by cubes with $5 \mu \mathrm{m}$ side lengths. In this context, they are usually attached to each other, forming a "dimer" with the $\alpha$ tubulin having the negatively charges end, as represented in Figure 4.

The dimers are connected end to end forming filaments for the wall of the microtubule. These filaments, however, are not strictly parallel but instead are coiled about the microtubule axis as represented in Figure 3 which shows from an end-view that there are 13 filaments creating a microtubule wall. The microtubules emerge from the centrosome, and increase in length up to a point where the end falls away, leaving the microtubules shorter. It then continues to emerge from the centrosome (the MTOC) and get longer once again. The "falling away" 


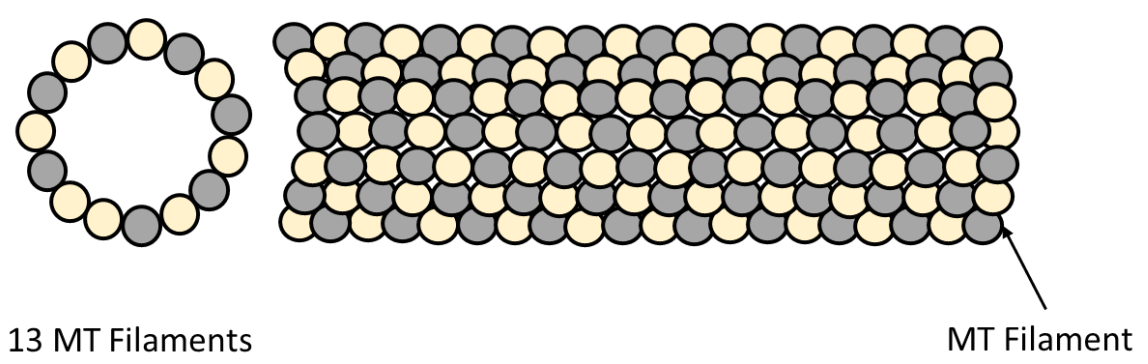

Figure 3. A microtubule model.

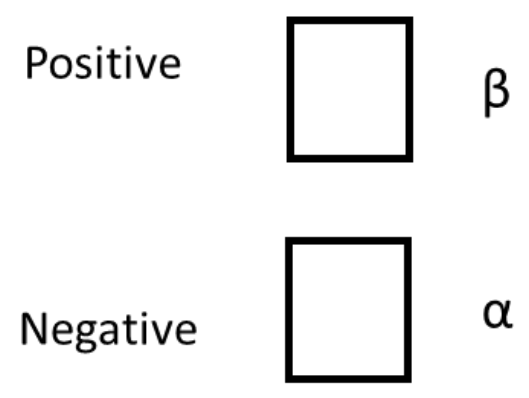

Figure 4. A Model of $\alpha$ - $\beta$ tubulin dimer.

is known as "catastrophy" whereas the relengthing is human as "rescue".

\section{DNA and the Chromosome}

The chromosome is a complement to the centrosome. The chromosome is composed of DNA (deoxyribonucleic acid) which can expand for extreme lengths (miles) and also contract to less than 1-nanometer thickness. When the DNA is fully contracted, it forms the mature chromosome ready for separation when the cell is about to divide. Figure 5 provides a representation of these organelles.

As seen in Figure 5, the chromosome has the approximate form of a capital "H", with four major arms, the connection of these arms is known as the "centromere". The "q-arms" are slightly longer than the "p-arms".

\section{Kinetochores}

The centromere is a source of microtubule-like strands known as "kinetochores". Indeed, the kinetochores are the analog of microtubules just as the chromosome is the analog of the centrosome.

Therefore, as the centrosome is also known as the microtubule organizing center (MTOC), the chromosome may well be known as the kinetochore organizing center "KTOC".

\section{Mitosis}

According to a dictionary definition: "mitosis" is a process that takes place in the nucleus of a dividing cell [6].

When a cell is about to divide, two self-duplication organelle activities occur: 


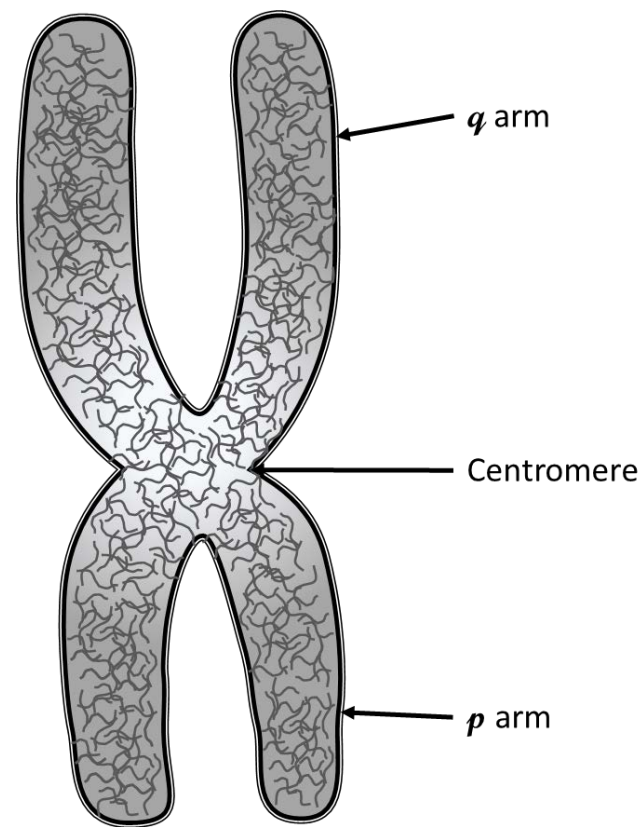

Figure 5. Representation of a chromosome.

1) the pair of centrioles self-duplicate into two pairs and 2) the DNA self-duplicates to double its volume.

Of all the organelles in a cell, only the centriole (in the centrosome) and the DNA (in the chromosome) have the ability to self-duplicate themselves and they do it essentially at the same time.

As illustrated in Figures 6-9, once the two pairs of centriole are created, they separate with the younger pair moving away along the boundary of the nucleus. It continues to follow the boundary until it reaches the diametrically opposite side of the nucleus.

Once the centrioles are established diametrically about the nucleus, they act like centrosomes, emitting microtubules onto the surface of the nucleus as represented in Figure 10.

At the same time, the chromosome emits kinetochores as represented in Figure 11.

With the microtubules emitted from the centrosomes and the kinetochores emitted from the chromosomes, their directions are perpendicular to each other. Taken together they form a perpendicular grid across the nucleus as represented symbolically in Figure 12. The vertical lines are the microtubules and the horizontal lines are the kinetochores. The kinetochores seek the microtubules and attempt to attach themselves to the microtubules. The centrosomes are then equal but opposite forces pulling the nucleus apart into two halves.

\section{Nucleus Response}

Just before the forces by the chromosomes tend to pull the nucleus apart, the nucleus itself softens and narrows itself in the center as represented by Figure 13. 


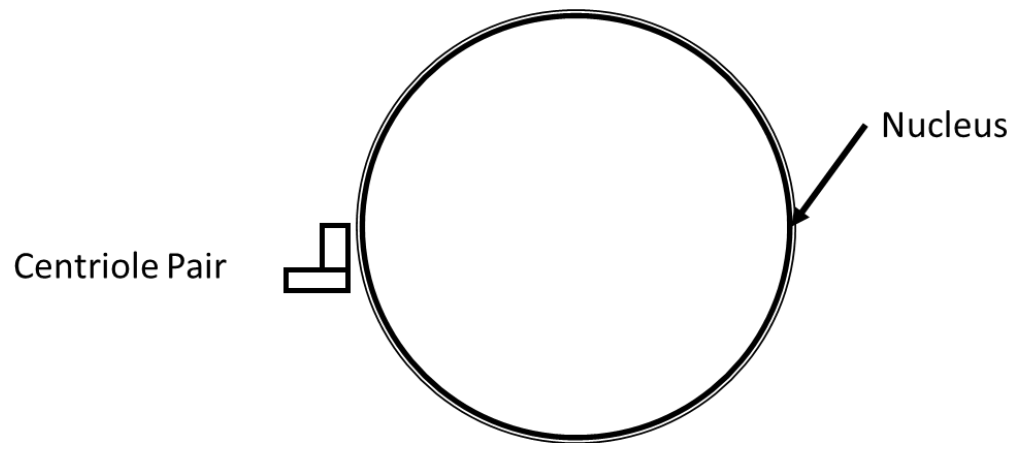

Figure 6. Centriole pair adjacent to the nucleus prior to mitosis.

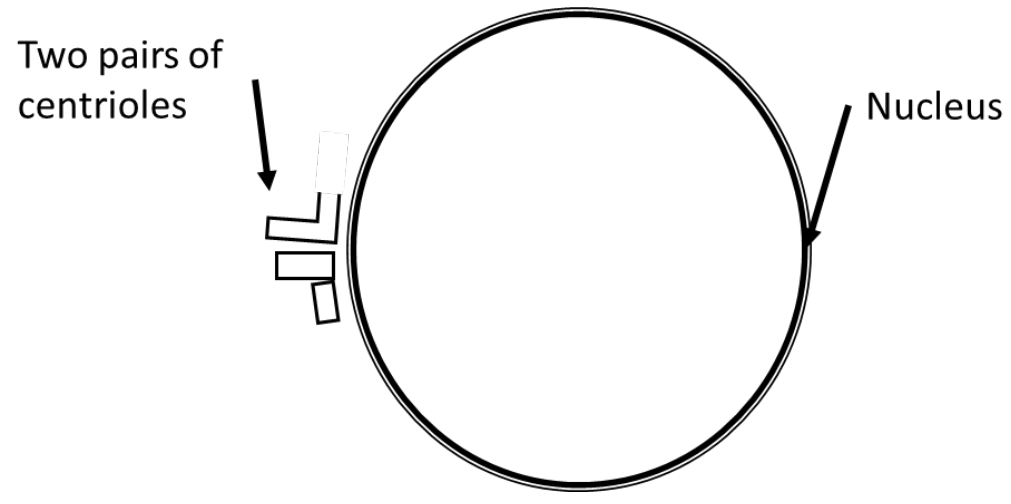

Figure 7. Original pair of centrioles divided into two pairs.

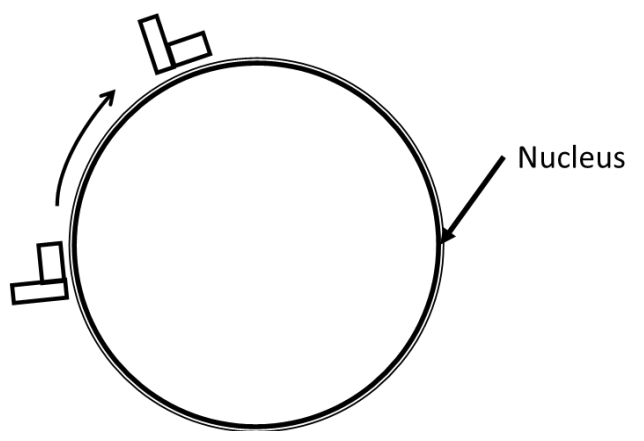

Figure 8. Younger pair of centriole migrating about the nucleus body.

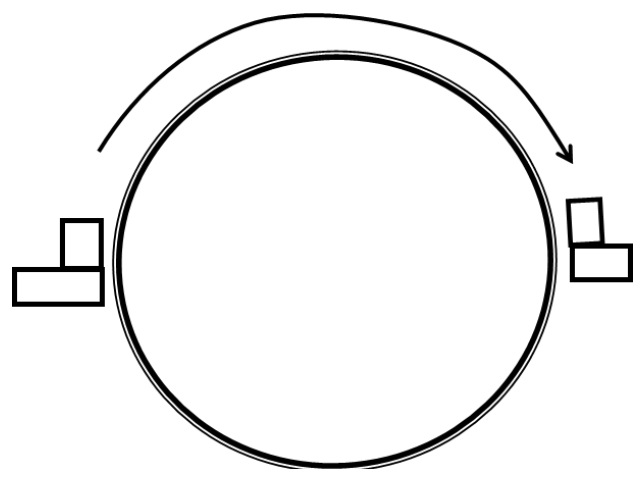

Figure 9. Younger pair of centriole reaching the diametrically opposite side of the nucleus. 


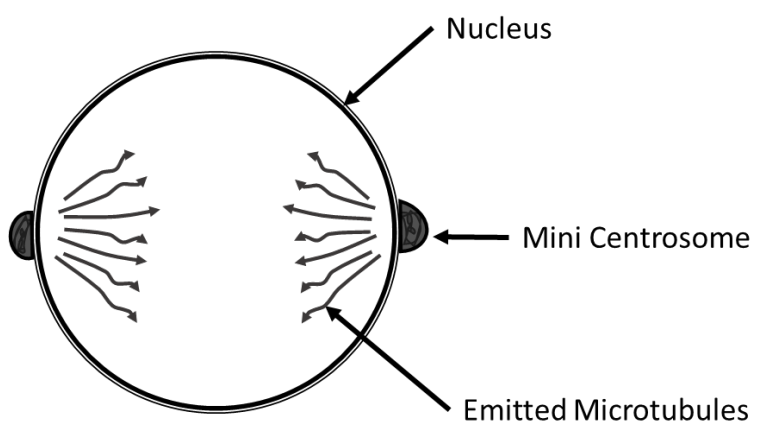

Figure 10. Microtubules extending about the nucleus.

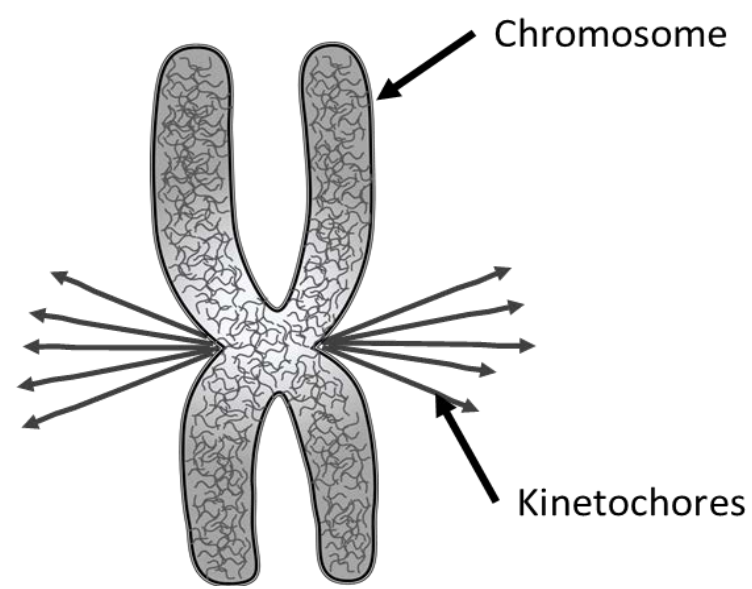

Figure 11. Chromosome emitting kinetochores.

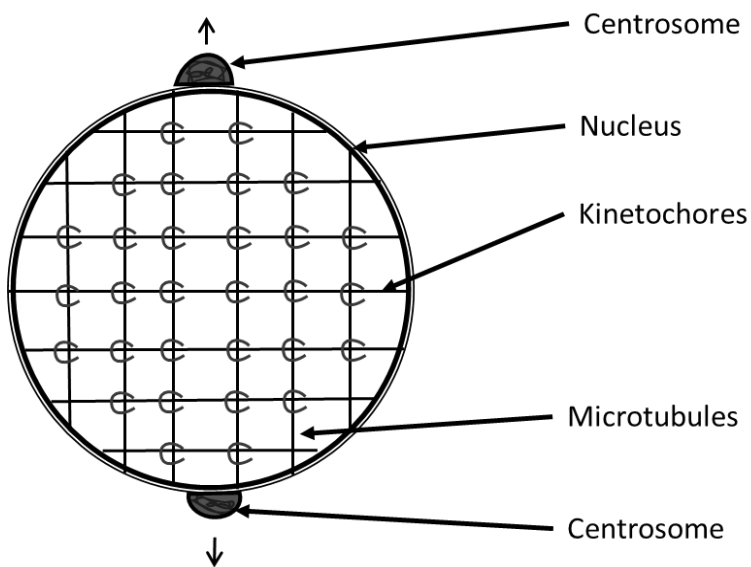

Figure 12. Symbolic representation of kinetochores attaching themselves to microtubules.

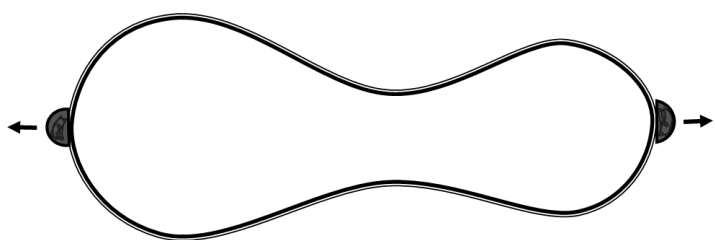

Figure 13. Nucleus response to opposing tension forces exerted by the centrosomes. 


\section{Cytoplasm Division and Concluding Remarks}

The cytoplasm is all the interior of a cell except for the nucleus. It is the cytoplasm which determines the cell type as requires by the body's organs, bones, and skin. When the cell is fully divided, or separated and duplicated into two identical cells, each takes with it half of the cytoplasm.

Finally, the foregoing description of cell division and duplication is devoted to normal cells-not cancer cells. Cancer cells may have multiple nucleus; therefore, the foregoing description would not be applicable to cancer cells. The principal difference is that cancer cells generally have more than two pairs of centrioles "supernumerary centrioles".

\section{Conflicts of Interest}

The authors declare no conflicts of interest regarding the publication of this paper.

\section{References}

[1] Albert, B., Bray, D., Lewis, J., Raff, M., Roberts, K. and Wilson, J.D. (1994) Molecular Biology of Cell. 3rd Edition, Garland Publishing, New York.

[2] Harden, J., Burton, G. and Kleinsmith, L.J. (2015) Becker's World of the Cell. 8th Edition, Pearson Publishing, New York.

[3] Sherman, I.W. and Sherman, V.G. (1983) Biology-A Human Approach. Oxford University Press, New York.

[4] Huston, R.L. (2016) A Review of Centriole Activity, and Wrongful Activity During Cell Division. Advances in Bioscience and Biotechnology, 7, 169-182. https://doi.org/10.4236/abb.2016.73015

[5] Huston, R.L. (2016) Mechanics of Centriole Microtubules. Advances in Bioscience and Biotechnology, 7, 266-277. https://doi.org/10.4236/abb.2016.76025

[6] Merriam-Webster Inc. (1983) Webster Ninth New Collegiate Dictionary, Springfield, MA., Merriam-Webster. 